\title{
Open bite - dental and skeleta. Differential diagnosis, prophylaxis and treatment. Retention problem
}

\author{
Petar Baev, L. Matev, Tz. Tonchev, Ch. Madjova, \\ A. Pomakova, M. Milkov \\ Medical University „Prof. Dr. Paraskev Stoyanov“ - Varna \\ Faculty of Dental Medicine
}

\begin{abstract}
Background: Open bite is a common and complex malocclusion. The etiological factors are well described and in most cases this malocclusion appears in childhood and it is due to deleterous habits. Not only can thumb-sucking cause this problem but also some upper and lower airways obstructions. Because of the obstruction the child cannot sleep normally and this may cause sleep apnea.

Aims: To describe the connection between different specialist such as orthodontists and otolaryngologist, which in most cases can diagnose this condition and work as a team.

Study Desigh: self-controlled study.

Methods: The following article is going to represent methods for diagnosting and treatment of dental and skeletal open bite malocclusion. It will be discussed the main etiological factors and different types of treatment in early, mixed and permanent dentition. Treatment plan can be orthodontic, orthodontic-surgical and the appliances used for this purposes can be removable and fixed. There are extraction and non-extraction methods and the benefits and disadvantages will be discussed.

Results: For perfect occlusion and normal airways we must first remove the etiological factors and then continue with orthodontics. If orthodontic treatment is firstly conducted, but the etiological factor such as hypertrophied palatal tonsils is not removed, we will not have a stable result and there is a risk of relapse.

Conclusion: Stable results can be accomplished with proper treatment, including variety of specialist, and long-enough period of retention.
\end{abstract}

Key words: sleep apnea, pre-orthodontic trainer, cephalometry
$\mathrm{O}$ pen bite occlusion belongs to the teeth-jaw deformities, which showed a lack of contact between the cutting edges of the upper and lower front teeth. Horoshilkina divide it into 3 levels - I - up to $5 \mathrm{~mm}$, II - between 5 and $9 \mathrm{~mm}$, III - more than $9 \mathrm{~mm}$. Posterior open-bite is rarely seen (premolars and molars). The frequency in mixed dentition is high and the prognosis of treatment depends on the severity of the disease. Open-bite is combined with Class I, Class II and Class III deformities. [1] This type of occlusion is characterized by Bjork with distal condylar inclination of the TMJ, short shoulder of the lower jaw, increased maxillary height, upright mandibular canal, thin and long symphysis of the lower jaw, abnormal occlusion plane and extruded molars, reduced interincisal corner (Fig. 1). [2] Some authors devided open-bite malo-



Figure 1. Bjork's features 
occlusion into 2 types - dental (the finger-sucking habit) during childhood impedes normal eruption of the anterior teeth and alveolar growth. The second type is sceletal form which is devided into 2 other forms. They represent viscerocranium with changed proportions. The viscerocranium includes the nasal bones, the vomer, upper and lower jaws. The first form represent clockwise rotation of the mandible with long maxilla. The second form shows sceletal open bite caused by skeletal deformation such as tipping of the maxilla and diversion of the gonial angle of the mandible (Ar-Go-Me). (Fig. 2). Magni-



Figure 2. Types of development of the viscerocranium

tude and duration of force application are important factors for the stability of tooth position. The occlusal contact and the eruptive or extrusive movement of the tooth determine the vertical position of the posterior teeth. The magnitude of tooth contact force on the posterior teeth depends on the strength of muscular activity. The duration of daily tooth contact varies between 1 and 3 hours, depending on the duration of mastication, swallowing, and sleeping. Unless the magnitude and duration of occlusal contact force are sufficient to prevent elongation of the posterior teeth, they will extrude, resulting in open bite. For example, the food features or the foodintake habits of young people, such as consumption of soft diet within a short eating time and irregular meal intake, could result in reduced tooth contact of the posterior teeth and subsequent development of vertical occlusal problems. Regular eating with appropriate mastication results in properly development of the inned and outter muscles layers of the oral cavity. [3]

The etiological factors of open-bite malocclusion is very important because this is the only way the dental orthodont can examine the patient and to make a treatment plan. The treatment plan includes prevention, orthodontic and orthognatic surgety.
The etiology of this type of malocclusion environmental factors and genetic factors. The environment factors iclude deleterious habits, mouth breathing and traumatism. The genetic factors include the way of growth and development of the facial structures.

The most important functional factors are - deleterious habits, thumb-sucking, anterior postition of the tongue, tongue thrust, mouth breathing, traumatism.

In a normal occlusion, there is a balanced relationship among the oral structures, basal bones, teeth, and intra and extraoral musculature, reflecting in a correct function of the stomatognathic system. This is denominated the buccinator mechanism. Thus, the teeth are in a balanced position receiving opposing forces arising internally by the tongue and externally by the lips and cheeks. Humans start sucking fingers, tongue, and lips during fetal life, in the maternal womb. At birth, the infant has a well-developed function of sucking to receive the nutrients essential for life. It is during suction developed in breastfeeding that the children not only get the nutrients that need to meet the physiological demands, as well as feelings of security, warmth, and acceptance necessary for their welfare and for their proper emotional development (Fig. 3). At this stage, suction is a mean of communication of the infant with the environment. The


Figure 3. Thumb-sucking as a deleterious habit 
early well-developed oral perception provides a sense of comfort, safety, and emotional satisfaction during sucking. When breastfeeding is not possible, the use of bottles with orthodontic nipples that resemble the anatomy of a woman's breasts is recommended, because they allow better contact of the tongue with the palate, as necessary for normal swallowing. When a child is bottle-fed, his physiological demand is met, but the natural need to suck is not supplied in the few minutes spent in the mother's lap. Thus, the child can begin the compensating thumb or pacifier sucking. Pacifier or thumb-sucking are considered as mechanisms of emotional supply of the child. Conse-


quently, pacifier or thumb-sucking in the early child development is considered normal. Through these habits, the child releases the emotional tensions from lack of affective care resulting from conflicting relationship between child and parents, which becomes a way to draw attention from people close to them. When children grow and develop other means of communication with the external environment, they usually spontaneously abandon the sucking habit. Interruption of sucking habits during the deciduous dentition can provide self-correction of the anterior open bite. However, persistence of the habit until the mixed dentition represents a deviation from normality, because these habits are potent etiologic malocclusion factors, particularly for anterior open bite. Deleterious oral habit does not always necessarily results in an open bite. First, it depends on how the habit is exercised, that is, it depends on the duration (for how long it is exercised, e.g., for how long the child keeps the pacifier in position), on the frequency (number of times per day it is exercised), and on the intensity

(the amount of force developed by the habit) of the habit. These factors are important in the etiology of this malocclusion and are known as Graber's Trident. The tongue is considered to have a secondary role in the etiology of anterior open bite because it can maintain or aggravate the existing open bite when placed between the anterior teeth. Patients can have macroglossy, infantile swallowing. Tongue thrust during swallowing is considered to be consequent to a previously established anterior open bite caused by deleterious oral habits. (Fig. 4) This is explained by the physiologic maturation of swallowing. The child without irrupted deciduous teeth presents an infantile


Figure 4. Tongue thrust

swallowing, which is normal at this stage. The characteristics of an infantile swallowing are separated jaws, active contractions of the musculature of the lips, tongue placed between the gum ridges, in contact with the lower lip, and little activity of the posterior tongue or pharyngeal musculature. Babys have the habit of infantile swallowing when there are no teeth but after 6 months they spontanously abandon this habit. (Fig. 5)

The Etiology of nasal obstruction can be devided into upper and lower breathing obstacles. The upper

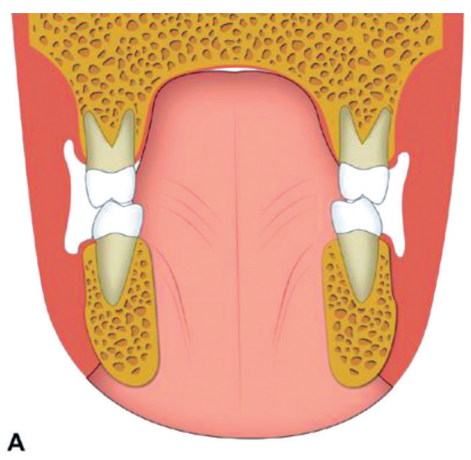

Figure 5. (A): Normal tongue position. (B): Lowered tongue position during mouth breathing.



(B): Lowered tongue position 
obstacles are hypertrophied adenoids (Fig. 6), allergic rhinitis, nasal turbinates hypertrophy and nasal septum deviation. The lower obstacles are hypertrophied or frequent tonsilitis (Watson 1981: LinderAronson et al. 1986). Adenoid hypertrophy is the abnormal growth of the adenoids, and in some situations, this growth is so exacerbated that can cause
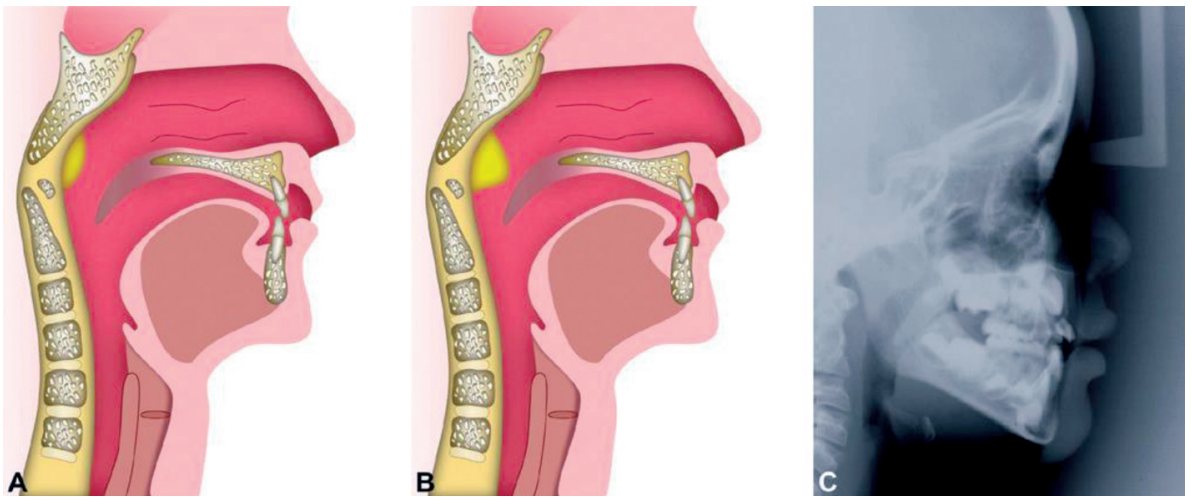

Figure 6. A): Normal adenoid; (B) and (C): hypertrophied adenoid partial or complete blockage of the air passage through the upper airways. There are two main ways to assess adenoid size: (1) indirect assessment with the nasopharyngeal mirror and (2) lateral headfilm. Two factors are crucial for the occurrence of blockage of the upper airways: (1) the size of the adenoids, and (2) the size of the nasal pharynx passageway. Generally, the adenoid reaches its largest size at 5 years of age, and then the growth is interrupted and adenoid atrophies in late childhood at about 7 years of age. Allergic rhinitis is a medical term describing irritation and acute or chronic inflammation of the nasal mucosa. The main symptoms of allergic rhinitis are rhinorrhea (exess nasal secretion), itching, and nasal congestion and obstruction (Sur and Scandale 2010). This is one of the main causes of nasal airway obstruction in the young child (Rubin 1980). The nasal septum is the part of the nose that seperates the two airways and the nostrils. A deviated septum is when there is a shift from the midline or center position. In normal condition, the nasal septum is centralized, and the air passages in the nasal cavity are symmetric. The nasal septum deviation is an abnormality in which a portion of the cartilaginous tissue deviates to one side of the nostril causing an obstruction for the airway passage on the side on which the deviation occured. The main etiological factors of nasal septum deviation are impact trauma, such as by a blow to the face and a congenital disorder, causing by compression of the nose during childbirth (Metson and Mardon 2013). The main symptoms of a deviated septum are infections of the sinus and sleep apnea, snoring, repetitive sneezing, facial pain, nosebleeds, and breathing difficulty.

Lower respiratoty obstacle is hypertrophied palatine tonsils. (Fig. 7). The tonsils are tho structures forming part of the lymphatic system that are located at the entrance of the upper airway and which has as main function to help the body's defence against respiratory infections. Tonsillitis is an infection of the tonsils that causes inflammation and represents the most common acute manifistation of tonsillar pathology. The main symptom of tonsillitis is sore throat, and the pain is usually worse then swallowing. Other symptoms can include fever, general ill feeling, headaches and vomitting. Tonsilar hypertrophy is the enlargement of the tonsils, but without the presence of inflammation. The obstructive tonsillar hypertrophy is the main indication for tonsillectomy. The main signs and symproms that may be observed in patients with tonsillar hypertrophy are sleep disturbances in a wide spectrum of severity, loud snoring, irregular breathing, coughing, night choking, interupted sleep apnea, dysphagia and excessive daytime sleepiness. To be able to breathe, the mouth-breathing child remains most of the time with the mandible in a lowered position to keep the mouth open. The tongue follows the mandible and consequently will not establish contact with the palate in the rest position, as usual, during nose breathing (Proffit et al. 2007). The absence of lat-

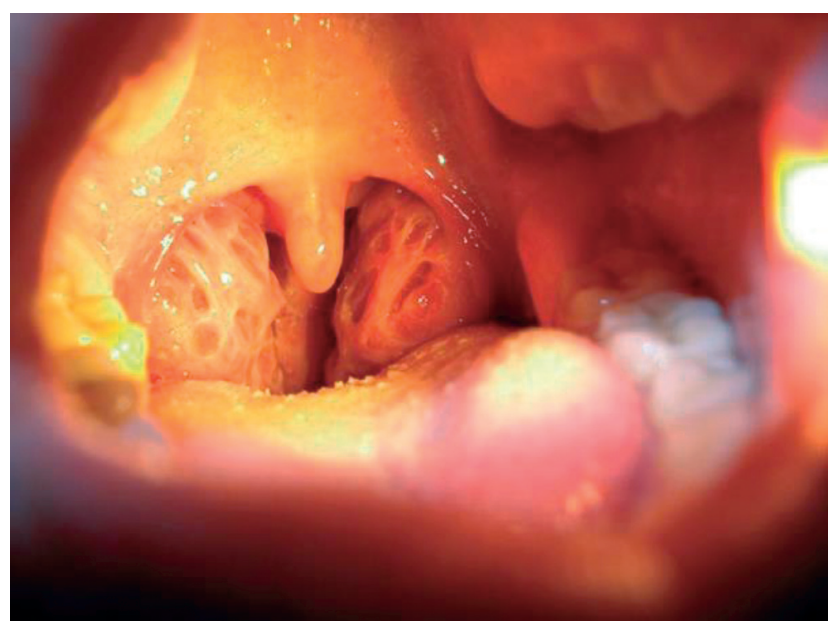

Figure 7. Tonsillitis causing lower airway obstruction due to enlarged palatine obstruction tonsils. 
eral contact of the tongue with the palate results in predominance of lingual forces of the bucinator muscle, and this consequently will result in smaller transverse development of the palate, which can cause posterior crossbite. Concurrently, there will be greater posterior vertical development of the palate. Besides, the tongue can rest over the anterior teeth, restricting their vertical development and contributing to anterior open bite. Genetic factors are growth pattern, craniofacial anomalies, cleft lip/palate. In orthodontics, patients can be classified into three groups according to their growth pattern: horizontal, normal, or vertical growth pattern. Some congenital deformities and syndromes can cause malocclusion, including anterior open bite. Cleidocranial dysostosis is a congenital deformity usually associated with heredity, which may be associated with the presence of anterior open bite. Treacher Collins syndrome involves hypoplastic mandible, glossoptosis,small size of the pharynx and nasopharynx, and occasionally choanalatresia can cause severe breathing problems, and consequently, open-bite malocclusion. Juvenile rheumatoid arthritis is a disease with oral involvement with anterior open bite, limited mouth opening, and in most of the cases, compromises the temporomandibular joint. [4]

Open bite malocclusion in the posterior region (Fig. 7 ) is observed in premolar and molar regions and in some cases there is exessive vertical growth of the mandible. A lateral open bite is occasionally seen in association with early extraction of first permanent molars, possibly occuring as a result of lateral tongie spread. There are two rare conditions which affect the eruption of the permanent buccal segment teeth. The first condition is primary failure of eruption. This condition almost exclusively affects molar teeth and is of unknown aetiology. Although bone resorption above the unerupted tooth proceeds normally, the tooth itselfs appears to lack any eruptive potential. Extraction is the only treatment alter-

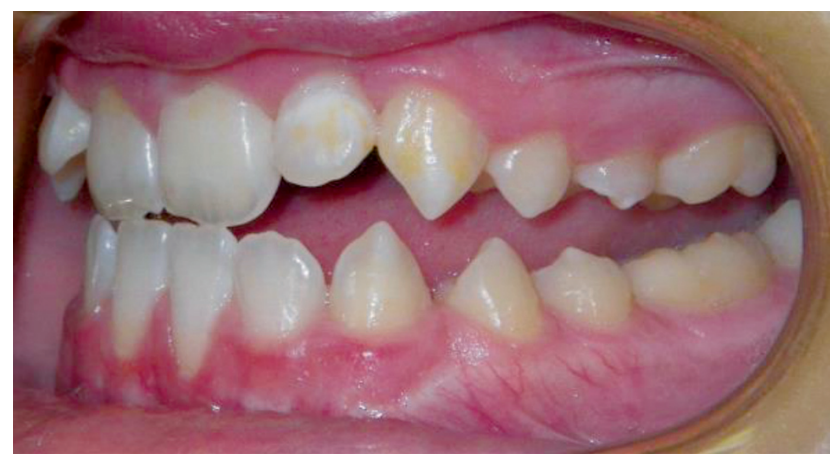

Figure 7. Posterior open bite native. The second condition is arrest of eruption. This usually involves molar teeth. Affected teeth appear to erupt normally into occlusion, but then subsequently fail to keep pace with occlusal development. As growth of the rest of the dentition and alveolar processes continues, lack of movement of the affected tooth or teeth results in relative submergence. The aetiology is ot understood and extraction is the usual treatment. More rarely, posterior open bite is seen in association with unilateral condylar hyperplasia which also results in facial asymmetry. Condylectomia is required. [5]

\section{Differential diagnosis}

There are two types of open bite malocclusion dental (Fig. 8) and skeletal (Fig. 9). The dental form is lighter than the skeletal and usually affects only the front teeth, the vertical dimension, the mandible angle is normal. There is no clockwise rotation of

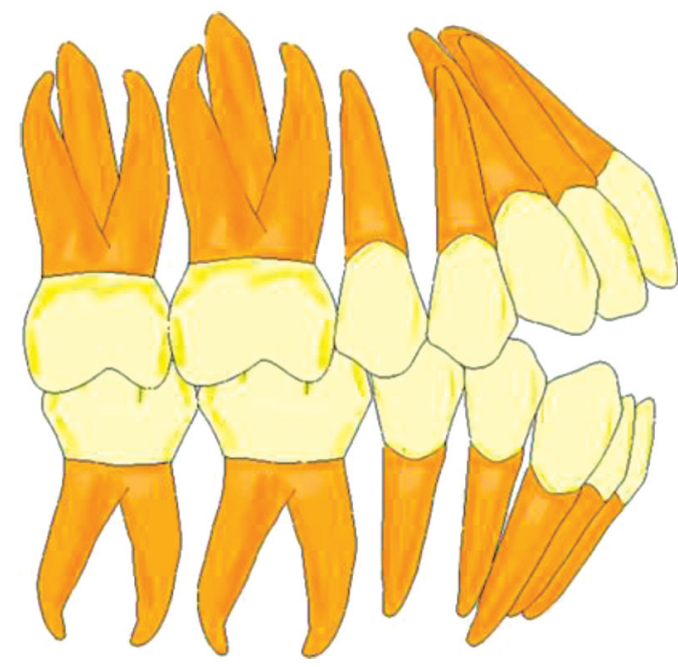

Figure 8. Dental form

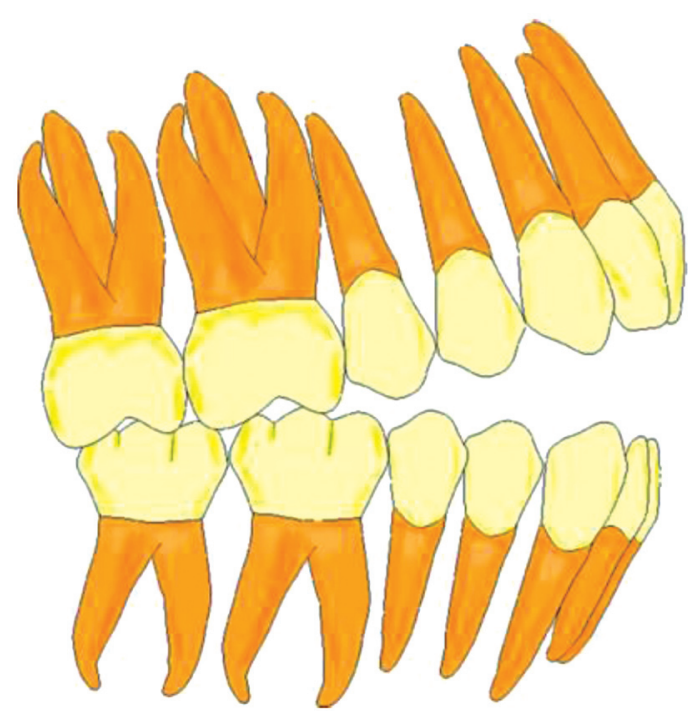

Figure 9. Skeletal form 
the mandible and there is no tension on the lips. The cephalometry of the skeletal form shows enlarged mandible angle (Ar-Go-Me), disparity of the facial skeleton with vertical enlargment of the down floor of the face, inclination of the hard palate, clockwise rotation of the mandible, tension on the lips. Premolars can be affected. The interlabial space is pathognomonic for skeletal affection. Nahoum says that it should be emphasized that cephalometrics, at best, is a descriptive technique which helps to define or classify a condition but does not necessarily provide us with the insight for the successful treatment of an anomaly. His index - N/ANS:ANS/Gn $=0.8$ reported vertical dimension of the skeletal with two types of rotation of the mandible- clockwise rotation and counterclockwise rotation of the mandible (hypodivergent and hyperdivergent types). He also reported reduced values of SN-PP angle at this type malocclusions, explaining it with the reduced height of the face due to anterior maxillary rotation. Correct diagnosis is important for the treatment, since dental forms most often need completely orthodontic treatment, while skeletal forms - orthodontic-surgical treatment. In orthodontic planning we need to observe whether we need to extrude the anterior teeth or to intrude the posterior teeth. The decison depends on the vertical position of the maxillary incisors to the lip-line. If front teeth don't show enought when smilling, we need to extrude the maxillary front teeth. Intrusion can be undertaken when the maxillary front teeth show enough when smilling. Although all of the skeletal characteristics just mentioned are associated with an anterior open bite, in one study (Dung $\mathrm{Dj}$, Smith $\mathrm{Rj}$ ) only 13\% of patients who had a cephalometric criterion for openbite tendency had a vertical space between the incisors perpendicular the occlusal plane. In many instances the skeletal open bite is camouflaged by overeruption of the anterior teeth. This issue makes classification of an open bite as either skeletal or dentoalveolar difficult. Often this malocclusion is the result of a combination of both factors. Skeletal open bites are often related to excessive vertical growth of the dentoalveolar complex, especially in the region of the posterior maxillary molar. (157. Conversely, dental anterior open bites are primarily due to reduced incisor dentoalveolar vertical. The difference between these two types of open bites is also reflected in the occlusal planes. The skeletal type of malocclusion generally has occlusal contacts only at the molar level, with both occlusal planes diverging anteriorly, whereas the occlusal planes in the dentoalveolar open bite usually diverge from the first premolar forward (Fig. 10). [6]



Figure 10. A-skeletal form, C-dental form

\section{Prophylaxis}

Prophilaxis is some kind of a part of the treatment. It is important because even with an exact treatment performed, if we do not unresolved etiological factor, relapse may occur. During the growth of the jaw, removing of deleterious habits may lead to a normalization of the discrepancies in dental forms. This is typical for the temporary dentition. Thus we remove the reasons that pressed or prevent the eruption of the frontal teeth. Using a pacifier of young parents is a good solution, because it flattens when used and it is removed as a habit easier than thumb-sucking (Fig. 11). Thumb-sucking and

Figure 11. Orthodontic pacifier




paciefiers must be removed until the age of 5. A child with tongue thrust must visit a orthodontic. Preventive orthodontic appliances (Fig. 12) provide

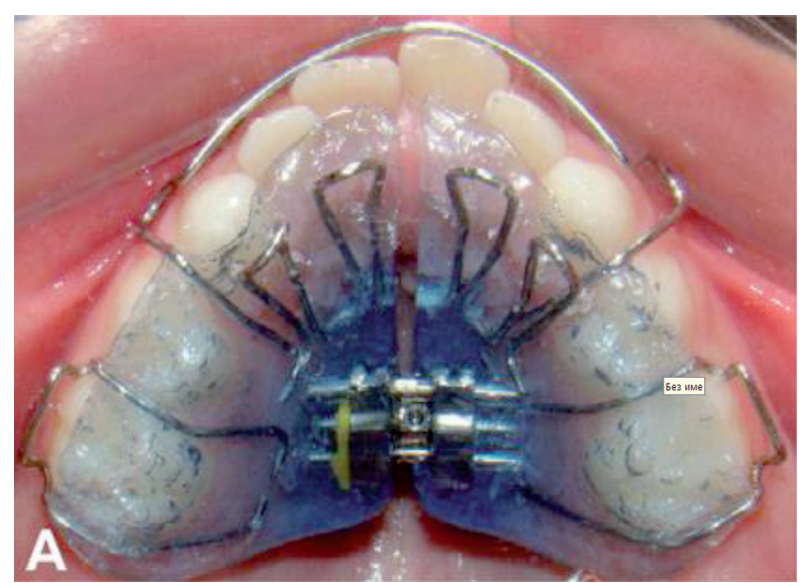

Figure 12. Removable palatal tongue crib

an opportunity for active primary and secondary prevention. They are used to remove bad habits that are etiologic factors for open bite occlusion. Apply in the period of temporary and mixed dentition. Some of these appliences are vestibular Kraus plate, palatal plate with barrier for the tongue, mandibular plate with barrier of Krumova for tongue thrust. Orthodontic treiners (Fig. 13) are the latest plates



Figure 13. Orthodontic trainer

for the period of early mixed dentition for open bite correction. They do not require impressions. Wear it one hour a day and during sleep. Lips must be folder when wearing except when talking. The child breath through the nose. No lip activity when swallowing, which allows the front teeth to develop correctly. They are clean by hot water after using. Two times a weak can be cleaned with solution of special tablet. The design consist of tooth channels and labial bows, which guide erupting teeth, tongue tag for correct tongue positioning, lip bumpers for discourage overeactive lip muscle activity. [7]

\section{Treatment}

In the temorary dentition the treatment is easier because in $95 \%$ of the cases there is observed dental form and very little prevalence of skeleta. Removing delaterious habits lead to a spontaneous self-correction fo the open bite. If this malocclusion is not removed at this stage the skeletal part increases. There is a period after removing the bad habit before treatment. If the child does not determinated the habit we must treat. Treatment plan in primary dentition is shown in mixed dentition.

In the period of mixed dentition the skeletal part increases and even if the patient remove the bad habit, self-correction is minimal. The orthodontist can wait for 6 months for self-correction before treatment. Worms and Kelly observed that if the open bite is 2 or more $\mathrm{mm}$ we must not wait, because self-correction cannot occur. Palatal plate with rings of the upper first molars and a barrier for the tongue shows great efficiency, but there is a period of adaptation in which the patient encounter problems with speech and eating. Removable appliances acts as reminder for the patints that he or she must not perform the bad habit. However, this gives some freedom of the child to remove the appliance and perform the habit. So that fixed devices are more effective. The tongue barrier of the palatal plate should be extended through the cervical part of the lower incisors with no conntact to the alveolar bone. The plate retrudes and extrudes the frontal teeth. Impressions are taken. The plastic behind front teeth is removed because

vertical growth appeared. The whole treatment in primary and mixed dentition varies from 3 to 18 months. During retention period Hawlay plate is recommended (Fig. 14). The modified Hawlay plate has occlusal stops of pos-



A



Figure 14. A - Modified Hawley plate with a tongue crib Hawley plate with a tongue crib and posterior bite; C - Hawley plate with an orifice in the incisive papillae region to help correct positioning of the tongue in the rest 
terior teeth, and the standart plate has a orifice for the papilla incisiva. Speech therapy can be added. Creepers for the tongue can be added for removing irregular tongue positioning (Fig. 15).


Figure 15. Tongue spurs can also be used in the mixed dentition.

In permanent dentition pravail the sceletal compoment. Treatment is more complex and requires more stability and retention. Fixed appliances are prefered. Elastics are used for extrusion of the upper front teeth. Closing an open bite in nonextraction treatment requires planning, beginning with bracket bonding. The anterior teeth should be bonded more cervically because this procedure will allow additional vertical movement of these teeth to extrude and consequently will help in closing the bite (Alexander 1983) (Fig. 16, 17). In particular, the mandibular anterior teeth have to be bonded more cervically because this will allow a greater overbite of the anterior maxillary teeth, working as an overcorrection. Leveling and alignment can

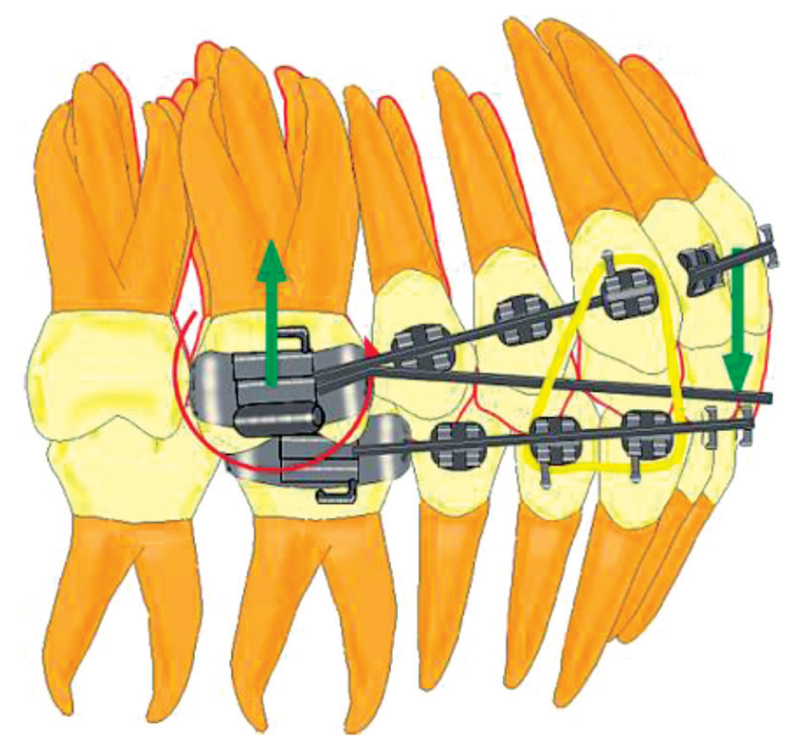

Figure 16. Fixed appliances with elastics for intrusion of the molars and extrusion of the incisors be obtained with different archwire sequences, following the usual principle of proceeding from the lightest to the heaviest archwire. It may begin with round nitinol wires, from the lightest to the heaviest, until a 0.016-in stainless steel archwire can be inserted. Vertical elastics are usually used when 0.018-in stainless steel archwires are placed. Otherwise, one can also conduct leveling and alignment with progressively increasing rectangular thermoactivated NiTi archwires until a $0.019 \div 0.025$-in archwire is inserted. Vertical elastics are then used with these archwires. All archwires should be flat, with no reversed or accentuated curve of Spee. If there are transverse discrepancies, these should be corrected before placing the vertical elastics to close the bite. The elastics are recommended to be used as much as possible, except during meals, which corresponds to approximately 18-20 hours of usage per day. If the patient complies with these guidelines, closure occurs at a rate of $1 \mathrm{~mm}$ per month. If no change in overbite is observed after 3 months, it is most likely that the patient is not correctly complying in using the elastics. There are several ways to check this. Failure to show up at the appointments with the elastics in place, inability to place the elastics, and no need for extra elastics are signs of lack of compliance. Ideally, once the bite is closed, the elastics still have to be used as recommended, for 4 months, which is the necessary time for bone to form in the alveolus (alveolar bone remodeling), because the anterior teeth extruded, coming off of the alveolus, to close the bite. After this period, daily use of the elastics should gradually decrease during 8 months; thereafter, the appliances can be removed. This is a period of active retention. However, the longer the active retention period, the greater will be the tendency for stability. Once fixed appliances are removed, a maxillary retention plate should be installed to be used 20



Figure 17. Cervically bonded brackets 
hours a day, except during meals. The maxillary retention plate may have the usual characteristics of a Hawley plate with an orifice in the region of the incisive papillae, which is mostly used. In some cases, extractions are necessary to correct other problems, such as crowding, excessive biprotrusion or anteroposterior discrepancies [8].

Extraction mechanics to close the open bite usually incorporates another important effect that helps to increase the overbite, which is the „drawbridge principle." (Fig. 18). This effect comes into action



Figure 18. Drawbridge principle

B



of vertical tooth intrusion necessary for malocclusion correction (Sherwood et al. 2002). When the intrusion force is applied only to the buccal or lingual surface of the tooth crown, the resultant force vector is away from the center of resistance of the tooth, generating an uncontrolled rotational moment that tends to tip the tooth toward the side that the intrusion force is acting. A skeletal anchorage with mini-implant or miniplate can be placed on each side of the maxillary or mandibular arch, such that the intrusion forces generate opposite rotational moments, which counterbalance each other, allowing an intrusionmovement with minimal or no undesirable side-effect (Fig. 19). The intensity of the rotational moment is represented by the product of the orthodontic force $(\mathrm{F})$ and the perpendicular distance



Figure 19A. Mini-implant anchorage placed on each side of the maxillary arch to produce opposite rotational moments, which counterbalance each other during intrusion mechanics, allowing vertical tooth movement with minimal or no undesirable buccolingual tipping.

(d) between the line of force and the center of resistance (CR), as shown in figure 20. Thus, the longer the moment arm (d), the greater the rotating effect. Another mechanical option consists of using transpalatal or lingual arches to stabilize the buccolingual tooth position during posterior teeth intrusion (Fig. 21). Teeth intrusion has been considered an orthodontic movement that can predispose to a greater degree of external apical root resorption 




Figure 20. Intrusion force passing buccally to the center of resistance of the maxillary molar. Consequently, a rotational moment is created, and the tooth crown tends to tip toward the orthodontic force if no mechanical resource is used to control this sideeffect.

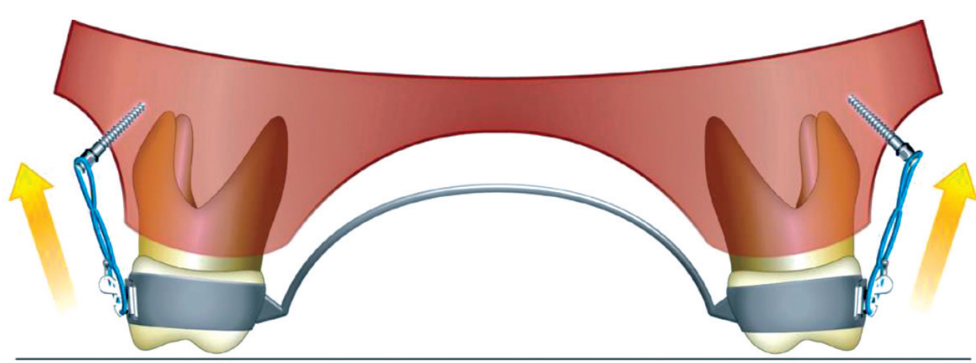

Figure 21. Transpalatal arch used to buccolingually stabilize molar position during

because intrusive forces produce located stress at the apex. Even when the intrusion force was smaller than $50 \mathrm{~g}$ for each tooth, external apical root resorption could be histologically shown. Successful molar intrusion can be achieved with a wide range of intrusion force values, which can vary from $100 \mathrm{~g}$ for each arch side to $300 \mathrm{~g}$ for each molar or two premolars. Although the intrusion force magnitude was not described, an intrusion force of 450 $\mathrm{g}$ is normal for treatment. With maxillary molar intrusion, there is consequent counterclockwise mandibular rotation and decrease in lower anterior face height (Fig. 22).

A different treatment protocol, including corticotomy (Fig. 23) and skeletal anchorage, has been proposed to reduce the intrusion time and root resorption degree. This protocol requires a subapical corticotomy procedure, which is performed before implantation of skeletal anchorage. The vertical cuts are made on the mesial side of the most anterior teeth and on the distal side of the most posterior teeth to be intruded. These cuts begin 2-3 $\mathrm{mm}$ above the alveolar crest and extend 5-6 $\mathrm{mm}$ beyond the apex where a horizontal cut is made to connect with two vertical cuts. This procedure is made on both the buccal and lingual sides so that the block of bone is retained only by the medullary bone. Sequentially, the skeletal anchorage is placed apically to the horizontal cut, and then an orthopedic intrusion force $(400 \mathrm{~g})$ is applied on the teeth contained in the bone block. This association between surface corticotomy and strong orthopedic intrusion force produces increased regional bone turnover (RAP-regional acceleratory phenomenon), which allows that an intrusion twice greater than that obtained without corticotomy is achieved in less than 2 months with minimal or undetectable root resorption because the bone blocks are moved together with the teeth. However, it must be considered that the corticotomy surgical procedure presents a significant greater biological cost, patient discomfort, and surgical risks than just miniimplant placement. Consequently, corticotomy disadvantages can justify patient acceptance for a



Figure $22 \mathrm{~A}, \mathrm{~B}, \mathrm{C}$. With maxillary molar intrusion, there is consequent counterclockwise



Figure 23. Corticotomy 
slightly increased intrusion time and a clinically nonsignificant tooth root shortening, mostly when open-bite severity does not require extreme molar intrusion. [10] [11]. Multisegmented maxillary osteotomy: This procedure is indicated in cases of skeletal anterior open bite that need transverse, anteroposterior, and superoinferior corrections, solving the problems in the three planes of space with a single procedure. Incision in the buccal sulcus is made from tuberosity to tuberosity followed by a LeFort I osteotomy. Interdental osteotomy is bilaterally performed between the maxillary lateral incisors and canines, extending to the nasal fossae, paramedially to the nasal septum, before lowering the maxilla (Fig. 24). The maxillary rectangular archwire is already segmented between the lateral incisors and canines. The segments will be


Figure 24. Cut lines for a multisegmented maxillary osteotomy.

immobilized, preserving the attached gingiva, the periodontal health, and the integrity of the palatal mucosa. It provides a better interdigitation than the one-piece LeFort I osteotomy and therefore allows greater postsurgical maxillary skeletal positioning stability, decreasing the chances for relapse. Generally, it is necessary for the patient to spend the first night at the hospital and be dismissed on the next day. For approximately a week, the patient should remain with intermaxillary blockage with several 3/16 medium-force elastics, keeping the mouth immobilized, or with insignificant movement, with a liquid diet. In the first postsurgical appointment, after a week, a lateral headfilm and a panoramic radiograph are taken to check the correct position of the condyles, plates, and screws. The surgeon removes all the elastics, allowing mouth opening and instructs on the use of only two or three 3/16 medium-force elastics, placed in square or trapezium shapes, bilaterally, oriented according to the type of surgery. This stage lasts approximately 8 weeks, with the patient using the elastics for 22 hours a day, removing them only during meals and hygiene. The diet can be pasty, turning to solid at the end. In the next appointment, after 9 weeks, the elastics are recommended to be used only during the sleeping hours, and exercises are prescribed for mouth opening, performed by the patient. Additionally, the patient is referred to the orthodontist for the final treatment stages. [12]

\section{Stability}

The retention devices are installed in the same appointment of appliance removal. The protocol is similar to conventional orthodontic patients, with the Hawley plate used continuously (removed only for meals) for 6 months and used only at night for the following 6 months. In the mandibular arch, retention is provided with a 0.024 -in stainless steel archwire from canineto- canine bonded retainer or, in case of initial accentuated crowding or rotations, a 0.0215-in Penta-One archwire canine-to-canine bonded retainer is used. The final records consist of a normal cephalometric headfilm, posteroanterior radiographs in cases of previous facial asymmetry, panoramic radiograph, final dental casts, and intra- and extraoral photographs. These records are essential to evaluate treatment stability and to show the obtained changes to the patient. [12]

\footnotetext{
References:

1. Moutavchiev, V Ортодонтия за общопрактикуващият стоматолог, София, 2003 стр.

2. Farhad B. Naini; Daljit S. Gill. Orthodontics - Principles and Practice 2011. Chapter 22, pages 217-220.

3. Nanda, R., BDS, MDS, PhD; Current Therapy in Orthodontics 2010. Chapter 14, pages 159-161.

4. Janson, G.; Valarelli, F.; Open-Bite Malocclusion Treatment and Stability 1st Edition 2014. Chapter 1, pages 1-22.

5. Mitchell, L.; An Introduction to Orthodontics 1996. Chapter 12, pages 127-128.

6. Nanda, R., BDS, MDS, PhD; Esthetics and Biomechanics in Orthodontics 2nd Ediotion 2014, Chapter 9 pages $147-150$.

7. Krumova, V.; Ръководство по ортодонтия за студенти. София, 2012, pages 114-120.

8. Janson, G.; Valarelli, F.; Open-Bite Malocclusion Treatment and Stability 1st Edition 2014. Chapter 2, pages $23-37$.

9. Janson, G.; Valarelli, F.; Open-Bite Malocclusion Treatment and Stability 1st Edition 2014. Chapter 3, pages $231-232$.

10. Janson, G.; Valarelli, F.; Open-Bite Malocclusion Treatment and Stability 1st Edition 2014. Chapter 4, pages 349-366.

11. Corticotomy facilitated orthodontics: Review of a technique - Prof. Ali Saad Thafeed AlGhamdi.

12. Janson, G.; Valarelli, F.; Open-Bite Malocclusion Treatment and Stability 1st Edition 2014. Chapter 5, pages 411-415.
} 